\title{
Electromagnetic Wave Interactions with 2D Arrays of Single-Wall Carbon Nanotubes
}

\author{
Taha A. Elwi and Hussain M. Al-Rizzo \\ Department of Systems Engineering, George W. Donaghey College of Engineering and Information Technology, \\ University of Arkansas at Little Rock, Little Rock, AR 72204, USA \\ Correspondence should be addressed to Taha A. Elwi, taelwi@ualr.edu
}

Received 13 June 2011; Revised 7 September 2011; Accepted 26 September 2011

Academic Editor: Raymond Whitby

Copyright ( ${ }^{2} 2011$ T. A. Elwi and H. M. Al-Rizzo. This is an open access article distributed under the Creative Commons Attribution License, which permits unrestricted use, distribution, and reproduction in any medium, provided the original work is properly cited.

\begin{abstract}
We report, for the first time, the scattering, absorption, and reflection characteristics of 2D arrays of finite-length, armchair, single-walled carbon nanotubes (SWNTs) in the visible frequency regime. The analysis is based on the Finite-Element-Method formulation of Maxwell's equations and a 3D quantum electrical conductivity function. Three geometrical models have been considered: solid cylinder, hollow cylinder, and honeycomb. We demonstrate that classical electromagnetic theory is sufficient to evaluate the scattering and absorption cross sections of SWNTs, which revealed excellent agreement against measurements without the need to invoke the effective impedance boundary conditions. The solid and hollow cylindrical models fail to provide accurate results, when both scattering and absorption are considered. Finally, it is shown that reflection and transmission characteristics of both individual and arrays of SWNTs, which are essential for solar cell applications, are strongly influenced by the length and the phenomenological parameters of the SWNT.
\end{abstract}

\section{Introduction}

Since arrays of SWNTs were introduced as trapping and absorbing layers to receive light from different angles, they became one of the most promising candidates for solar cell technology [1]. It is well known that the efficiency of solar cells decreases when the incident light deviates away from the normal to the cell's surface where a mechanical rotary system is required [2]. Therefore, enormous research efforts have been devoted to place SWNTs on top of photovoltaic cells to maintain efficient performance even when the sun is not directly overhead [3]. This is important for spacecraft applications to avoid mechanical aiming systems with minimum feature sizes and improved reliability [2].

The proper configuration of SWNT arrays, in terms of spacing and orientation, enables localization of light with minimum reflection and scattering losses, which is essential for increasing the conversion efficiency of solar cells [4]. For example, ultra-low refractive index films based on vertical SWNT arrays leading to reduced reflectance from solar cell substrates were recently demonstrated $[5,6]$.
SWNTs, which possess a ballistic conductivity, transport electrical charges over at least micrometer lengths [7]. One of the most celebrated theories on the ballistic conductivity of SWNT is the $\pi$-electron, tight-binding quantum conductance, and the effective impedance boundary conditions (EIBC) [8], which were subsequently used to evaluate the electromagnetic (EM) interaction with carbon nanotubes [9-16]. The classical Hallén-type integral equation was invoked to solve the dipole antenna problem and evaluate the scattering cross section $\left(\sigma_{\text {sca }}\right)$ from an individual SWNT $[9,10]$. The current distribution on an infinitely long carbon nanotube antenna fed by a delta-gap generator is reported in [11] using a Fourier transform technique. In [10], a model consisting of infinite 2D arrays of finite-length SWNT was presented to calculate the scattering cross section $\left(\sigma_{\text {sca }}\right)$ based on a periodic Green's function. In [13], the Leontovich-Levin equation was formulated to evaluate scattering by a carbon nanotube of finite length, both numerically and analytically. In [14], the $\sigma_{\text {sca }}$ of an infinite 2D array of SWNTs was studied using periodic Green's function and the Born approximation. In [15], the normalized $\sigma_{\text {sca }}$ was calculated in the Rayleigh 


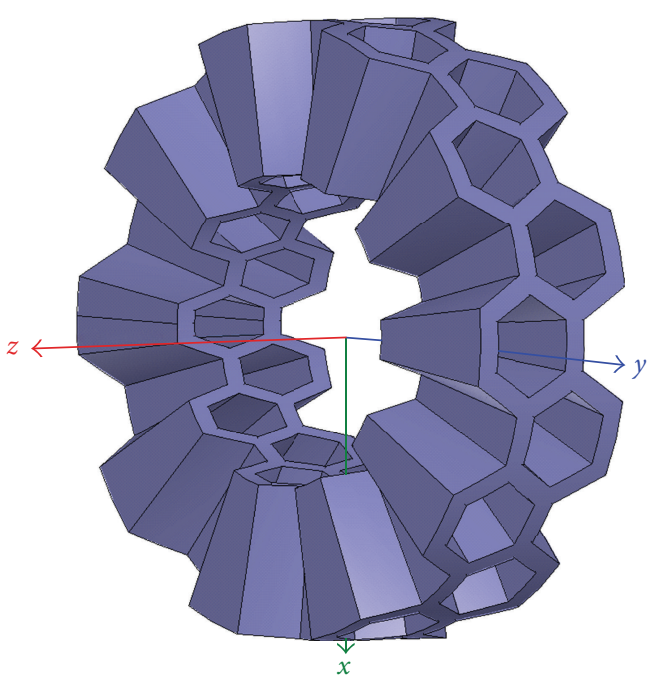

(a)

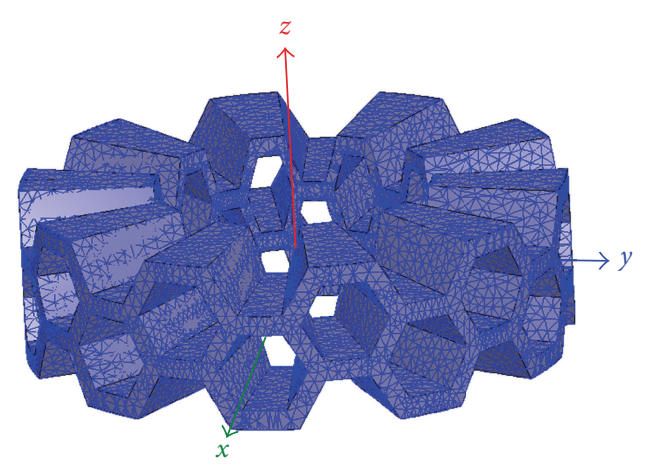

(c)

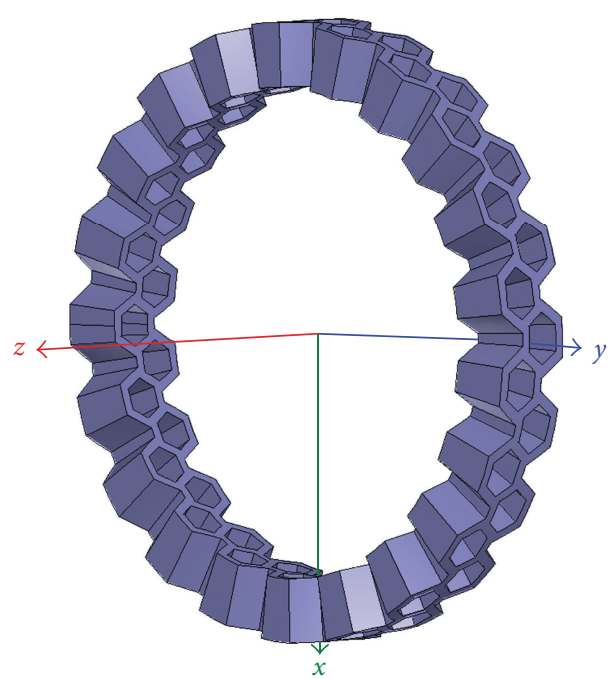

(b)

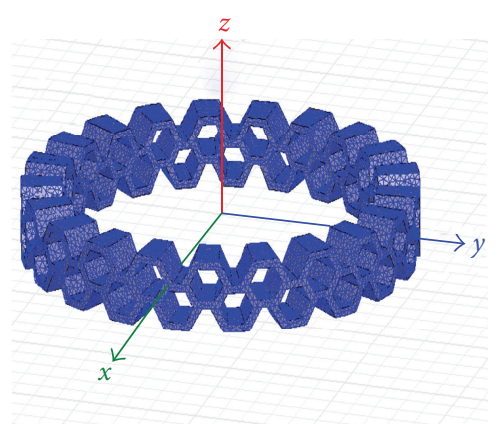

(d)

FIGURE 1: The numerical models of the HS for $(10,10)$ and $(21,21)$ armchair SWNT: (a) and (b) 3D geometrical models for $(10,10)$ and $(21,21)$ of SWNT; (c) and (d) are the mesh view for $(10,10)$ and $(21,21)$ of SWNT.

region based on a closed-form expression derived for an infinitely long, hollow cylindrical (HC) SWNT model of zero thickness and compared to experimental results. In [16], the absorption cross section ( $\left.\sigma_{\text {abs }}\right)$ was calculated for an individual and bundles of parallel, finite-length SWNTs. The analyses were based on the Fredholm integral equation of the first kind in the near-field region at different orientations and locations from the EM sources.

Two assumptions were common among previously reported studies [8-16]. First, the SWNT is modeled as a solid wire or as a thin cylindrical shell, both of which do not provide realistic values for $\sigma_{\text {abs. }}$. Second, only the axial surface current has been considered for the solid and hollow model. It should be noted that the phenomenological parameters in the research reported in [9-16] were determined based on comparing the calculated $\sigma_{\text {sca }}$ versus measurements without considering $\sigma_{\mathrm{abs}}$.

To the best of the authors' knowledge, we consider for the first time a realistic honeycomb-shaped (HS) model of two armchair SWNTs using 3D full wave numerical simulations to evaluate the EM interactions with SWNT in single and array configurations without invoking the EIBC. The $\sigma_{\text {sca }}$ and $\sigma_{\mathrm{abs}}$ of $(10,10)$ and $(21,21)$ SWNTs obtained from the HS, SC, and HC models are compared against the measured results published in $[17,18]$. Next, the reflection spectra from 2D arrays of SWNTs are considered for the SC, HC, and HS models in both horizontal and vertical orientations. Finally, the reflection spectra from the $(10,10)$ SWNT arrays are provided at different lengths of the HS model in both horizontal and vertical orientations.

\section{Theoretical Treatment}

We start from the ballistic, 1D quantum conductance model of SWNT based on the $\pi$-electron tight-binding quantum conductance defined below [8]

$$
\begin{aligned}
& \sigma_{c n}(\omega) \\
& =\frac{j e^{2} \omega}{\pi^{2} \hbar a}\left\{\frac{1}{\omega(\omega-j v)} \sum_{s=1}^{m} \int_{1^{\mathrm{s}} B Z} \frac{\partial F_{c}}{\partial p_{z}} \frac{\partial \varepsilon_{c}}{\partial p_{z}} d p_{z}\right. \\
& \left.\quad+2 \sum_{s=1}^{m} \int_{1^{\mathrm{st} B Z}} \varepsilon_{c}\left|R_{\gamma c}\right|^{2} \frac{F_{c}-F_{v}}{\hbar^{2} \omega(\omega-j v)-4 \varepsilon_{c}^{2}} d p_{z}\right\},
\end{aligned}
$$




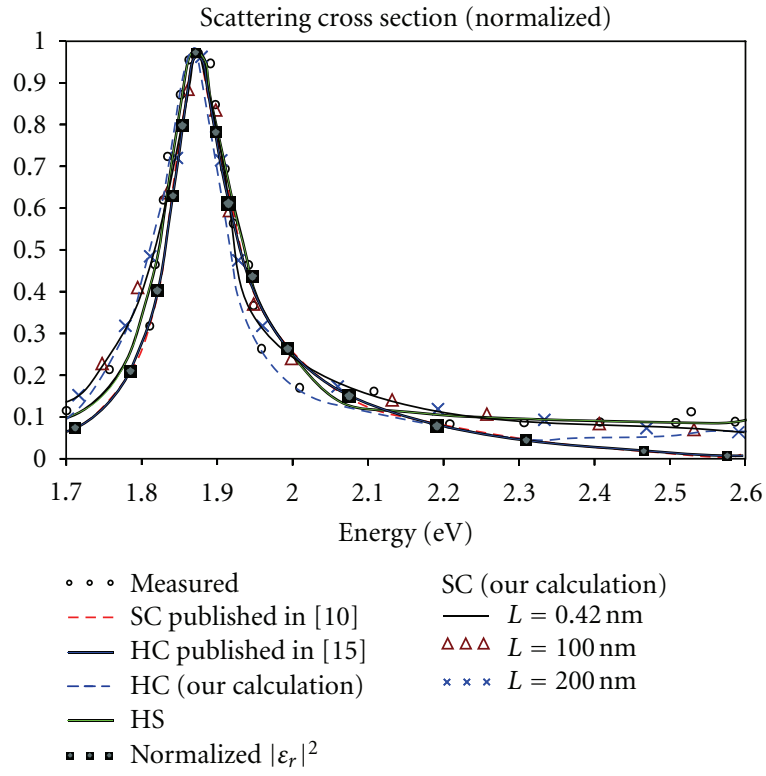

FIgure 2: The $\sigma_{\text {sca }}$ of SC, HC, and HS models of a SWNT compared against measured data in $[10,15]$.

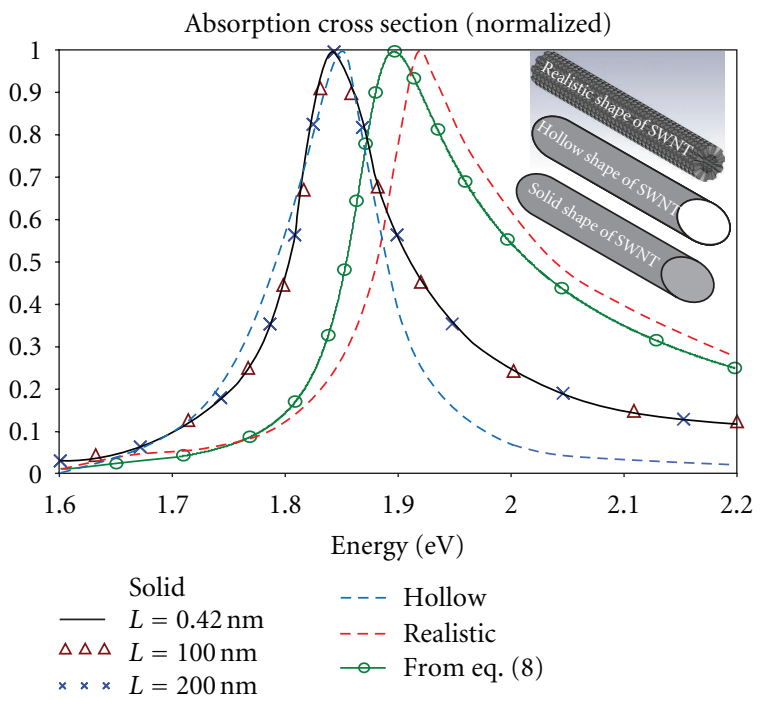

FIgURE 3: Comparison of $\sigma_{\mathrm{abs}}$ of the armchair $(10,10)$ SWNT for three different geometries.

where $v=\tau^{-1}$ is the relaxation frequency, $\hbar$ is the normalized Planck's constant, $1.05457 \times 10^{-34} \mathrm{~J} . \mathrm{s}, e$ is the electron charge, $1.602 \times 10^{-19} \mathrm{C}, \varepsilon_{c}$ and $F_{c, v}$ are the Fermi dispersion and electron dispersion relation function of the carbon nanotube, respectively, and $R_{v c}$ is the matrix element for the SWNT.

The 1D conductance of (1) is converted to a 3D conductivity $\left(\sigma_{3 d}\right)$ in $(\mathrm{S} / \mathrm{m})$ to properly represent the $3 \mathrm{D}$ SWNT model in our simulations. The impedance of a solid cylindrical conductor is given by [19]

$$
Z_{i}=\frac{\gamma_{i} J_{o}\left(a \gamma_{i}\right)}{2 \pi a \sigma_{3 d}(\omega) J_{1}\left(a \gamma_{i}\right)}
$$

where $a$ is radius, $a=0.678 \mathrm{~nm}$ for $(10,10)$ and $a=$ $1.424 \mathrm{~nm}$ for $(21,21), \omega$ is the radian frequency, and $\gamma_{i}$ is the skin depth, given by [10]

$$
\gamma_{i}=(1-j) \sqrt{\frac{\omega \mu \sigma_{3 d}}{2}} .
$$

Using the asymptotic forms of Bessel functions of the first and second order for small arguments, (2) can be approximated as

$$
Z_{i}=\frac{1}{\pi a^{2} \sigma_{3 d}(\omega)}
$$

The expression of the impedance per unit length of a SWNT is given by [11]

$$
Z_{c n}=\frac{1}{2 \pi a \sigma_{c n}(\omega)}
$$

Taking the ratio $\left(Z_{i} / Z_{c n}\right), \sigma_{3 d}$ can be determined from $\sigma_{c n}$ defined in (1) using the following formula

$$
\sigma_{3 d}(\omega)=\frac{2}{a} \sigma_{c n}(\omega) .
$$

In our numerical analysis, we used the complex relative permittivity to compute $\sigma_{\mathrm{sca}}$ and $\sigma_{\mathrm{abs}}$ of the individual SWNTs. It can be shown that the real and imaginary parts of the relative permittivity $\left(\varepsilon_{r}\right)$ can be expressed as

$$
\begin{aligned}
& \varepsilon_{r}^{\prime}=1+\frac{\sigma_{r}^{\prime}}{\omega \varepsilon_{o}}, \\
& \varepsilon_{r}^{\prime \prime}=\frac{\sigma_{r}^{\prime \prime}}{\omega \varepsilon_{o}},
\end{aligned}
$$

where $\sigma_{r}^{\prime}, \sigma_{r}^{\prime \prime}, \varepsilon_{r}^{\prime}$, and $\varepsilon_{r}^{\prime \prime}$ are the real and imaginary parts of the conductivity and permittivity, respectively.

\section{Comparison of Model Results with Measurements}

The $\sigma_{\text {sca }}$ of $(10,10)$ and $(21,21)$ armchair SWNTs are evaluated from Ansoft's High Frequency Structure Simulator (HFSS) [20] and compared to the measured data reported in [17] and [18], respectively. The SWNT is surrounded by an air box, the six faces of which are set as perfectly matched layer (PML) [21]. The incident field is a plane wave of unity amplitude, and the magnetic field is perpendicular to the SWNT axis similar to the measurement setup reported in $[17,18]$.

The complex relative permittivity of each geometry is generated from (7) by adjusting the phenomenological parameters $(\gamma$ and $\tau)$ in (1) in order to match the $\sigma_{\text {sca }}$ results generated from HFSS to the measured $\sigma_{\text {sca }}$ reported in $[17,18]$ for the $(10,10)$ and $(21,21)$ SWNTs, respectively. The numerical model of the HS is implemented in HFSS by building a single graphene layer from 20 honeycomb cells for the $(10,10)$ SWNT and from 42 honeycomb cells for the $(21$, 21) SWNT that is folded around the $z$-axis. The numerical 3 D models of the $(10,10)$ and $(21,21)$ SWNTs are presented 


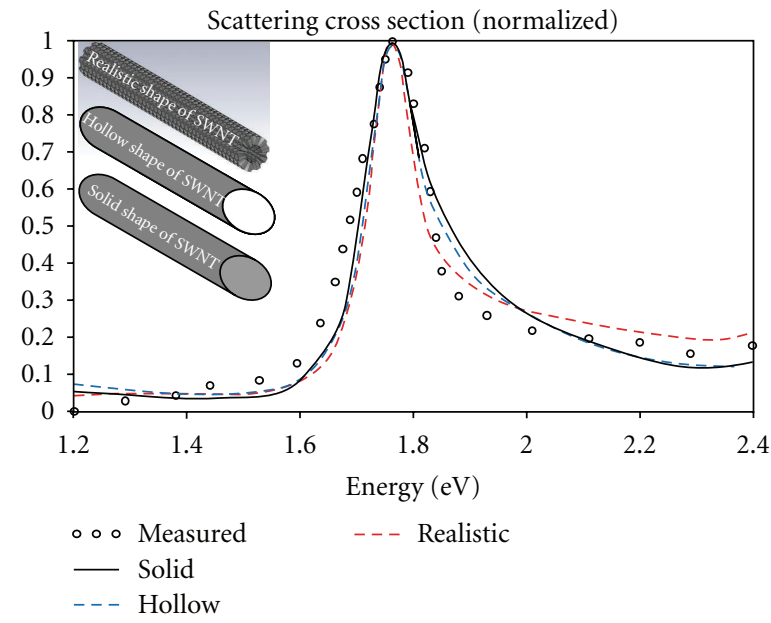

(a)

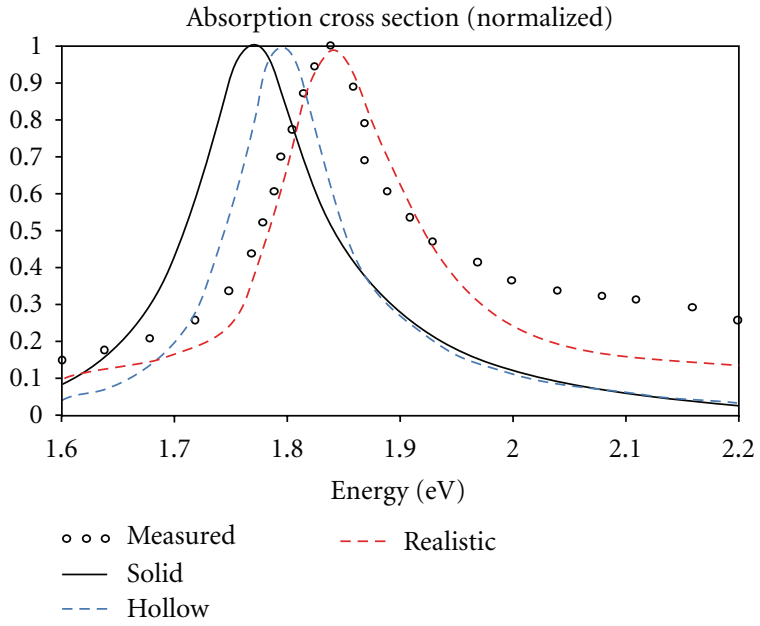

(b)

FIGURE 4: $\sigma_{\text {sca }}$ and $\sigma_{\text {abs }}$ of the armchair $(21,21)$ SWNT for three different geometries (SC, HC, HS) compared to measured results in [18]: (a) $\sigma_{\mathrm{sca}}$ and (b) $\sigma_{\mathrm{abs}}$.

in Figures 1(a) and 1(b), respectively. Results presented in this paper are based on the convergence criterion that the relative deviation of the computed $\sigma_{\mathrm{sca}}$ and $\sigma_{\mathrm{abs}}$ between at least two consecutive mesh passes should be below 2 percent. The mesh chosen is tetrahedral with typical mesh distributions shown in Figures 1(c) and 1(d) for the $(10,10)$ and $(21,21)$ SWNTs, respectively.

\subsection{Phenomenological Parameters from the Scattering Cross} Section. To estimate $\gamma$ and $\tau$ for the SC, HC, and HS models of the SWNT, HFSS is used to simulate $\sigma_{\text {sca }}$ of the $(10,10)$ SWNT, which is compared to the measured results reported in [17]. Next we compare $\sigma_{\text {sca }}$ for the three models to demonstrate the effect of the geometry on the estimated $\gamma$ and $\tau$. By varying $\gamma \approx 2.5-3.1 \mathrm{e} . \mathrm{V}$ and $\tau \approx 0.005-0.015 \mathrm{ps}$, we calculated the constitutive parameters of the respective SWNT geometry. We started by simulating the SC since both $\gamma$ and $\tau$ are reported in [10] to validate our computational procedure. Figure 2 displays the normalized $\sigma_{\text {sca }}$ for the (10, 10) SC armchair SWNT for three different lengths: $0.42 \mathrm{~nm}$, $100 \mathrm{~nm}$, and $200 \mathrm{~nm}$, along with the results reported in [10] and those measured in [17]. It can be seen from Figure 2 that the profile of the normalized $\sigma_{\mathrm{sca}}$ is independent of length. This is explained in $[10,14]$ based on the quantum effects associated with interband transitions.

However, the independence of the $\sigma_{\text {sca }}$ profile on length can also be described in terms of the classical Rayleigh scattering theory, which is applicable when the maximum linear dimension of the object is much smaller than the wavelength of the minimum frequency considered, as can be seen from the results depicted in Figure 2. In this case, $\sigma_{\text {sca }}$ is proportional to the oscillating dipoles induced inside the object. The oscillatory dipole moments are proportional to the electric permittivity of the object [22]. The normalized $\sigma_{\text {sca }}$ for both the HC and HS models of a SWNT $0.42 \mathrm{~nm}$ in length and $0.678 \mathrm{~nm}$ in radius are also presented in Figure 2. For the HC-shaped SWNT, the extracted $\gamma$ and $\tau$ are
TABLE 1: Comparison of $\gamma$ and $\tau$ against the published values for the three models (SC, HC, and HS) for $(10,10)$ SWNT.

\begin{tabular}{lcc}
\hline Model & Our calculated values & Published values \\
\hline SC & $\gamma=3.03 \mathrm{e} . \mathrm{V}, \tau=0.01 \mathrm{ps}$ & $\gamma=3.01 \mathrm{e} . \mathrm{V}, \tau=0.01 \mathrm{ps}$ \\
& & {$[10]$} \\
HC & $\gamma=3.05 \mathrm{e} . \mathrm{V}, \tau=0.0097 \mathrm{ps}$ & $\gamma=3.035 \mathrm{e} . \mathrm{V}, \tau=0.0097 \mathrm{ps}$ \\
HS & $\gamma=3.059 \mathrm{e} . \mathrm{V}, \tau=0.011 \mathrm{ps}$ & {$[15]$} \\
\hline
\end{tabular}

compared to those published in [15]. Results from simulated $\sigma_{\text {sca }}$ of the HS model are also shown in Figure 2.

It should be noted that the normalized $\sigma_{\text {sca }}$ reported in $[10,15]$ can be simply obtained from the normalized value of $\left|\varepsilon_{r}\right|^{2}$ as described in [22]. Therefore, results presented in $[10,15]$ can be simply obtained by normalizing the square value of the modulus of the complex permittivity versus energy, as can be seen in Figure 2. At low frequencies, $\sigma_{\text {sca }}$ obtained from the modulus of complex permittivity is closer to the measured results than at high frequencies. Moreover, this approximation is valid only in the Rayleigh region. However, when using HFSS, the calculated results produce better agreement with the measured $\sigma_{\text {sca }}$ for the three geometrical models and, furthermore, can be extended well above the Rayleigh region.

3.2. Absorption Cross Section. In general, the simulated $\sigma_{\text {sca }}$ for the three geometrical models provided an excellent agreement with the measured results reported in [17]. However, each individual model exhibits different $\gamma$ and $\tau$ as seen in Table 1 . These differences in $\gamma$ and $\tau$ and the geometrical model of the SWNT strongly influence the computed $\sigma_{\text {abs }}$ spectra as depicted in Figure 3.

In Figure 3, we present the spectrum of $\sigma_{\mathrm{abs}}$ computed by HFSS for the SC model with different lengths: $0.42 \mathrm{~nm}$, 


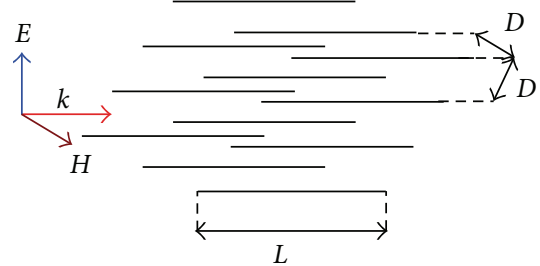

(a)

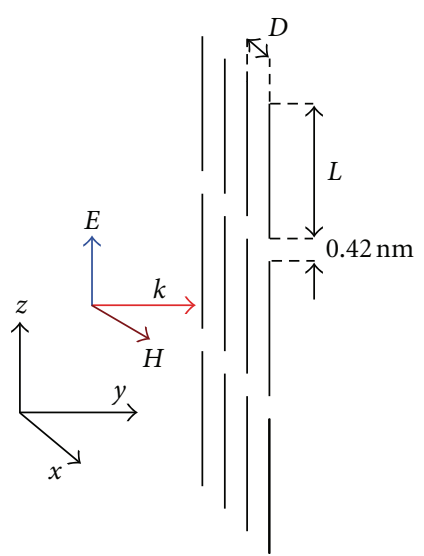

(b)

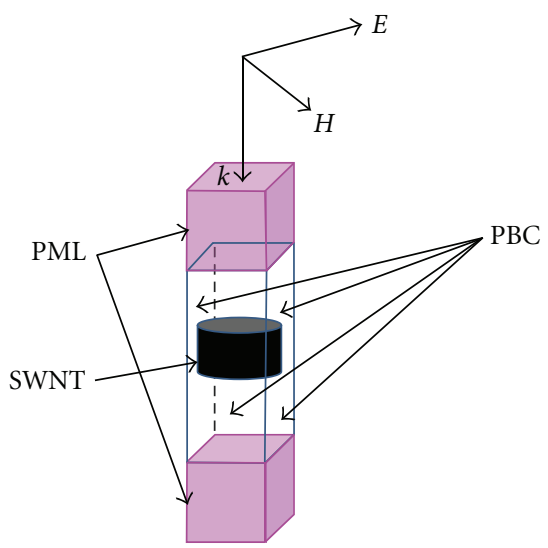

(c)

Figure 5: Alignments of the infinite 2D arrays of finite length SWNT; (a) vertical alignment, (b) horizontal alignment, and (c) the numerical setup of the 2D arrays of SWNTs as implemented in HFSS.

$100 \mathrm{~nm}$, and $200 \mathrm{~nm}$, and for both HC, and HS models computed from the following expression [16]:

$$
\sigma_{\mathrm{abs}}=\text { Normalized }\left[\frac{8 \pi^{2} a L}{c} \operatorname{Re}\left(\sigma_{c n}\right)\right] \text {, }
$$

where $L$ is the length of the SWNT and $c$ is the speed of light in vacuum. The normalized $\sigma_{\mathrm{abs}}$ is not very sensitive to the length of the SWNT, as is seen in Figure 3. The effect of the geometry of the SWNT is revealed in Figure 3 when comparing the $\sigma_{\mathrm{abs}}$ of the SC, $\mathrm{HC}$ and HS models. This observation reveals the geometrical dispersion introduced by the honeycomb shape of the SWNT, which is not captured by the SC and HC models. In Figure 3, it is observed that

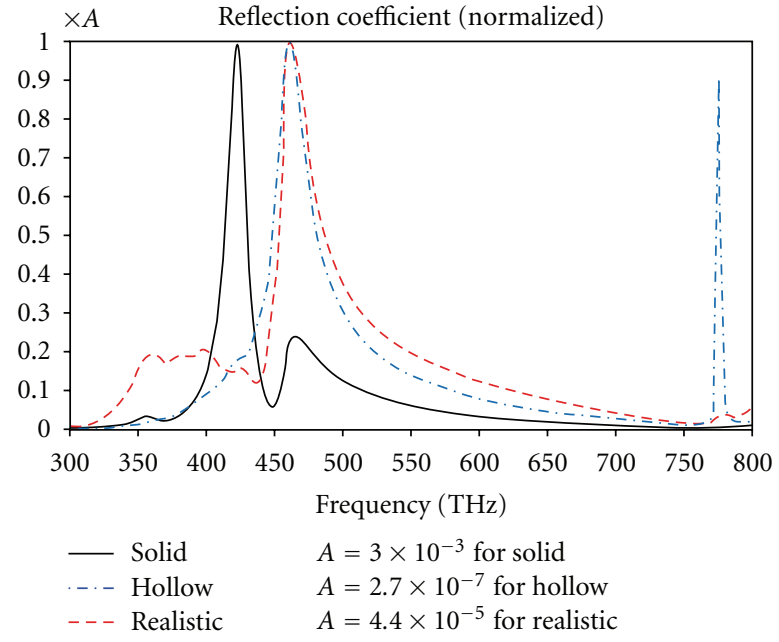

(a)

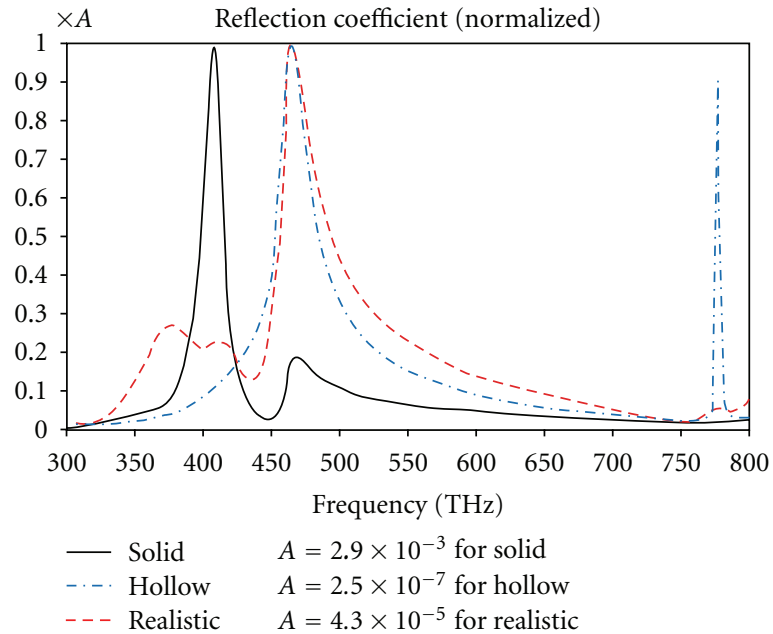

(b)

Figure 6: The reflection spectra of the 2D arrays of armchair (10, 10) SWNT using three geometrical models (SC, HC, and HS); (a) for vertical arrays and (b) for horizontal arrays.

$\sigma_{\mathrm{abs}}$ peaks at different frequencies for the three models. The spectral characteristics of $\sigma_{\mathrm{abs}}$ are different too.

Due to the differences observed in Figure 3, we performed additional studies to emphasize the effects of the geometrical model on the absorption characteristics of SWNTs. The measurements of the $\sigma_{\text {sca }}$ and $\sigma_{\text {abs }}$ reported in [18] for the armchair SWNT of index $(21,21)$ are used to further confirm the observations inferred from Figure 3. We started by simulating $\sigma_{\text {sca }}$ to find $\gamma$ and $\tau$ by using the same approach outlined in the previous section. The measured and simulated spectra of $\sigma_{\text {sca }}$ and $\sigma_{\mathrm{abs}}$ for the $(21,21)$ armchair SWNT are presented in Figures 4(a) and 4(b), respectively.

\section{Numerical Analysis for the 2D Arrays of SWNT}

For the numerical results presented in this section, we used the complex permittivity for the $(10,10)$ armchair SWNT 


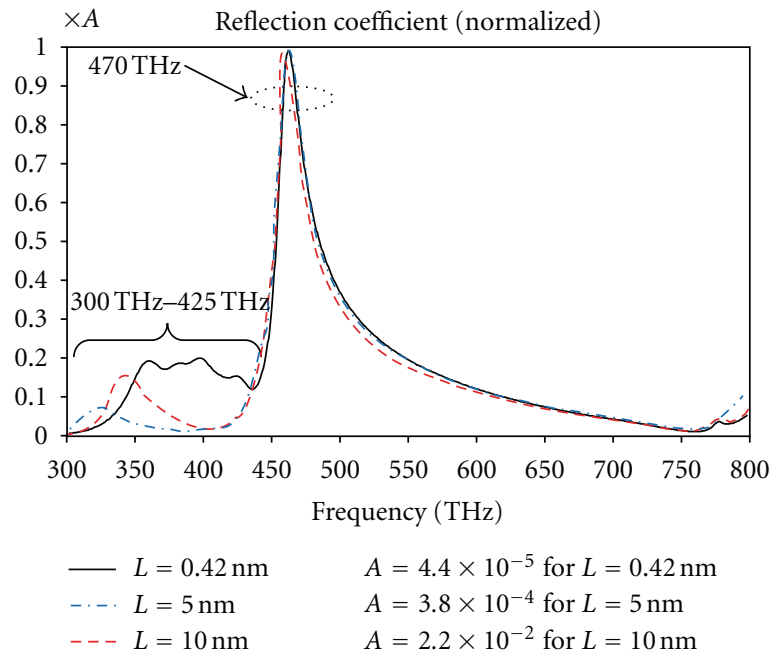

(a)

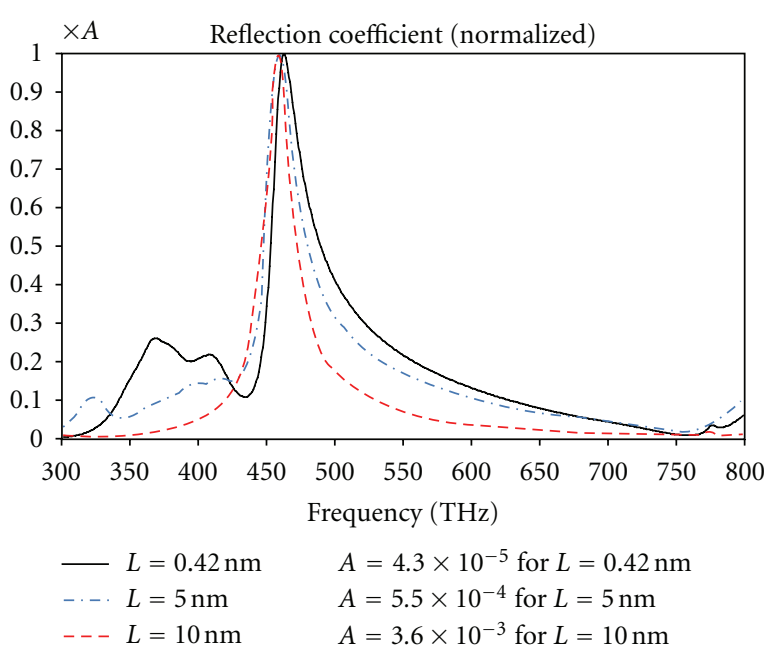

(b)

FIgURE 7: The reflection spectra of the 2D arrays of armchair $(10,10)$ SWNT of HS shape: (a) for vertical arrays and (b) for horizontal arrays.

developed in Section 2. The infinite array of finite-length SWNTs situated in the $x-z$ plane and the simulation setup used in HFSS are depicted in Figure 5. Two orientations of the SWNT array are considered. Vertical orientation is defined when the array is normal to the $x-z$ plane as seen in Figure 5(a). Horizontal orientation refers to the case in which the array is parallel to the $x-z$ plane as shown in Figure 5(b). The direction of propagation of the incident field is set to be normal to the $x-z$ plane as illustrated in Figures 5(a) and 5(b). The SWNTs are separated by a distance $D=2 a+0.34 \mathrm{~nm}$ $\approx 1.7 \mathrm{~nm},(0.34 \mathrm{~nm}$ corresponds to the Van der Waal's gap [22]) with three lengths, $L=0.42 \mathrm{~nm}, 5 \mathrm{~nm}$, and $10 \mathrm{~nm}$. Short carbon nanotubes have been chosen since the synthesis of arrays of short carbon nanotubes is much easier than with long carbon nanotubes [23]. Furthermore, long carbon nanotubes are vulnerable to bending, and the cost and time for growing short carbon nanotube are much less than for long tubes [24]. The 2D arrays of SWNTs are considered to be infinite in extent; however, for practical purposes, the dimensions of SWNTs arrays are finite. Since the length of an individual SWNT is much smaller than the arrays' dimensions, the diffraction effects from the arrays' edges can be ignored [14]. Following the standard procedure for the modeling of infinite periodic arrays of arbitrarily shaped scatterers as outlined in [21], an air box is used to surround the scatterer, and the four faces parallel to the direction of propagation are set as periodic boundary conditions (PBC). The remaining two faces are assigned as PML as shown in Figure 5(c).

Results are presented first for vertical and horizontal SWNT arrays with $D=1.7 \mathrm{~nm}$ and $L=0.42 \mathrm{~nm}$ for the SC, HC, and HS models to demonstrate the effect of the geometry on the reflection characteristics. Next, the effects of the length and orientation of the SWNT on the performance of arrays based on the HS model are reported. In general, it is found that the maximum reflection from the SWNT arrays occurs at frequencies ranging from $400 \mathrm{THz}$ to $470 \mathrm{THz}$ as depicted in Figure 6 . The simulated reflection coefficients are normalized by a constant, $A$. The magnitude of $A$ is presented in Figure 6 with the normalized reflection coefficients for the vertical and horizontal arrays. It can be seen that maximum reflection occurs for the SC model and the minimum for $\mathrm{HC}$ model. This is because the volumetric density of the SC model is much larger than the volumetric density of the HC model. It is found that for the two alignments, the effect is observed only on the magnitude of the reflection coefficient.

Next, the effect of length of the HS SWNT model on the performance of the SWNT arrays is investigated. For solar cells applications, the intrinsic thickness of SWNT layers may vary up to $50 \mathrm{~nm}$, and the separation distance should be very small such as to maximize the quantum efficiency [25]. Therefore, in our simulations we assumed $L$ to range from $0.42 \mathrm{~nm}$ to $10 \mathrm{~nm}$ and $D=1.7 \mathrm{~nm}$. The reflection coefficients for vertical and horizontal alignments are presented in Figure 7.

It can be seen that the reflection coefficients of the SWNT array decrease when the length of SWNT increases. However, the bandwidth and resonant frequency are not affected for all cases considered. Therefore, the reflection of light from arrays consisting of short SWNTs is less than that from arrays with long SWNTs. This is because the volumetric losses increase as the length of SWNTs increases. The bandwidth of modes excited from $300 \mathrm{THz}$ to $425 \mathrm{THz}$ decreases when the length of the SWNTs increases. The bandwidth around $470 \mathrm{THz}$ decreases when the length of the SWNTs increases only when the SWNTs are aligned horizontally as seen in Figure 7(b). Also, we found that the reflection coefficients for the SWNT arrays are proportional to the complex relative permittivity.

\section{Conclusion}

In this paper, the fundamental properties of EM interactions with SC, HC, and HS 3D models of SWNTs, both individual 
and in infinite array configurations, are investigated in the visible frequency range using the finite-element formulation of Maxwell's equations combined with a quantum conductivity function. The scattering and absorption cross sections of two armchair SWNTs are examined. It is found that only the HS model is capable of providing accurate results for the spectrum of both $\sigma_{\text {sca }}$ and $\sigma_{\mathrm{abs}}$ as compared against measured results. As far as SWNT arrays are concerned, maximum reflection is observed for frequencies ranging from $400 \mathrm{THz}$ to $470 \mathrm{THz}$, which occurs for the SC model, whereas the minimum is observed for the HC model. For the horizontally and vertically aligned SWNTs, only the magnitude of the reflection coefficient has been affected. The reflection of light from arrays consisting of short SWNTs is found to be less than that from arrays with long SWNTs. The bandwidth of the excited modes decreases when the length of the SWNTs increases. The bandwidth decreases as the length of the SWNTs increases when the SWNTs are aligned horizontally. Finally, the reflection coefficients for the SWNT arrays are proportional to the complex relative permittivity.

\section{Acknowledgments}

The authors would also like to thank Dr. Said M. Mikki and Dr. M.V. Shuba for their valuable discussions and comments. Finally, the authors thank Dr. George W. Hanson for his helpful discussions.

\section{References}

[1] Y. Jia, P. Li, X. Gui et al., "Encapsulated carbon nanotubeoxide-silicon solar cells with stable 10\% efficiency," Applied Physics Letters, vol. 98, no. 13, Article ID 133115, 2011.

[2] X. Dang, H. Yi, M. H. Ham et al., "Virus-templated selfassembled single-walled carbon nanotubes for highly efficient electron collection in photovoltaic devices," Nature Nanotechnology, vol. 6, no. 6, pp. 377-384, 2011.

[3] H. Zhou, A. Colli, A. Ahnood et al., "Arrays of parallel connected coaxial multiwall-carbonnanotube-amorphous- silicon solar cells," Advanced Materials, vol. 21, no. 38-39, pp. 3919-3923, 2009.

[4] I. A. Levitsky, "Hybrid solar cells based on carbon nanotubes and nanoporous silicon," IEEE Nanotechnology Magazine, vol. 4, no. 4, Article ID 5599964, pp. 24-25, 2010.

[5] H. Zhou, A. Colli, T. Butler et al., "Carbon nanotube arrays for optical design of amorphous silicon solar cells," International Journal of Material Forming, vol. 1, no. 2, pp. 113-116, 2008.

[6] Y. Wang, K. Kempa, B. Kimball et al., "Receiving and transmitting light-like radio waves: antenna effect in arrays of aligned carbon nanotubes," Applied Physics Letters, vol. 85, no. 13, pp. 2607-2609, 2004.

[7] S. Reich, C. Thomsen, and J. Maultzsch, Carbon Nanotubes: Basic Concept and Physical Properties, John Wiley and Sons, Hoboken, NJ, USA, 3rd edition, 2004.

[8] G. Y. Slepyan, S. A. Maksimenko, A. Lakhtakia, O. Yevtushenko, and A. V. Gusakov, "Electrodynamics of carbon nanotubes: dynamic conductivity, impedance boundary conditions, and surface wave propagation," Physical Review B, vol. 60, no. 24, pp. 17136-17149, 1999.

[9] G. W. Hanson, "Fundamental transmitting properties of carbon nanotube antennas," IEEE Transactions on Antennas and Propagation, vol. 53, no. 11, pp. 3426-3435, 2005.
[10] J. Hao and G. W. Hanson, "Infrared and optical properties of carbon nanotube dipole antennas," IEEE Transactions on $\mathrm{Na}$ notechnology, vol. 5, no. 6, pp. 766-775, 2006.

[11] G. W. Hanson, "Current on an infinitely-long carbon nanotube antenna excited by a gap generator," IEEE Transactions on Antennas and Propagation, vol. 54, no. 1, pp. 76-81, 2006.

[12] J. Hao and G. W. Hanson, "Electromagnetic scattering from finite-length metallic carbon nanotubes in the lower IR bands," Physical Review B, vol. 74, no. 3, Article ID 035119, pp. 1-6, 2006.

[13] G. Y. Slepyan, M. V. Shuba, S. A. Maksimenko, and A. Lakhtakia, "Theory of optical scattering by achiral carbon nanotubes and their potential as optical nanoantennas," Physical Review B, vol. 73, no. 19, Article ID 195416, pp. 1-11, 2006.

[14] J. Hao and G. W. Hanson, "Optical scattering from a planar array of finite-length metallic carbon nanotubes," Physical Review B, vol. 75, no. 16, Article ID 165416, pp. 1-7, 2007.

[15] S. M. Mikki and A. A. Kishk, "Theory of optical scattering by carbon nanotubes," Microwave and Optical Technology Letters, vol. 49, no. 10, pp. 2360-2364, 2007.

[16] M. V. Shuba, S. A. Maksimenko, and G. Y. Slepyan, "Absorption cross-section and near-field enhancement in finite-length carbon nanotubes in the terahertz-to-optical range," Journal of Computational and Theoretical Nanoscience, vol. 6, no. 9, pp. 2016-2023, 2009.

[17] M. Y. Sfeir, T. Beetz, F. Wang et al., "Optical spectroscopy of individual single-walled carbon nanotubes of defined chiral structure," Science, vol. 312, no. 5773, pp. 554-556, 2006.

[18] F. Wang, D. J. Cho, B. Kessler et al., "Observation of excitons in one-dimensional metallic single-walled carbon nanotubes," Physical Review Letters, vol. 99, no. 22, Article ID 227401, pp. 1-4, 2007.

[19] R. W. P. King and T. T. Wu, "The imperfectly conducting cylindrical transmitting antenna," IEEE Transactions on Antennas and Propagation, vol. AP-14, no. 15, pp. 524-534, 1966.

[20] "Ansoft's High Frequency Structure Simulator HFSS," 13th version, 2011, http://www.ansoft.com .

[21] D. Perry, "HFSS- antennas arrays and FSS'," Ansoft, http:// www.ansoft.com/hfworkshop02/perry.pdf.

[22] L. Tsang, J. A. Kong, and K. Ding, Scattering of Electromagnetic Waves, John Wiley and Sons, New York, NY, USA, 2000.

[23] H. Li and K. Banerjee, "High-frequency analysis of Carbon Nanotube interconnects and implications for on-chip inductor design," IEEE Transactions on Electron Devices, vol. 56, no. 10, pp. 2202-2214, 2009.

[24] M. S. Dresselhaus and G. Dresselhaus, Carbon Nanotubes: Synthesis, Structure, Properties and Applications, Spring, New York, NY, USA, 2001.

[25] M. Vaezzadeh, M. R. Saeedi, T. Barghi, and M. R. Sadeghi, "The necessary length of carbon nanotubes required to optimize solar cells," Chemistry Central Journal, vol. 1, no. 1, article 22, 2007. 

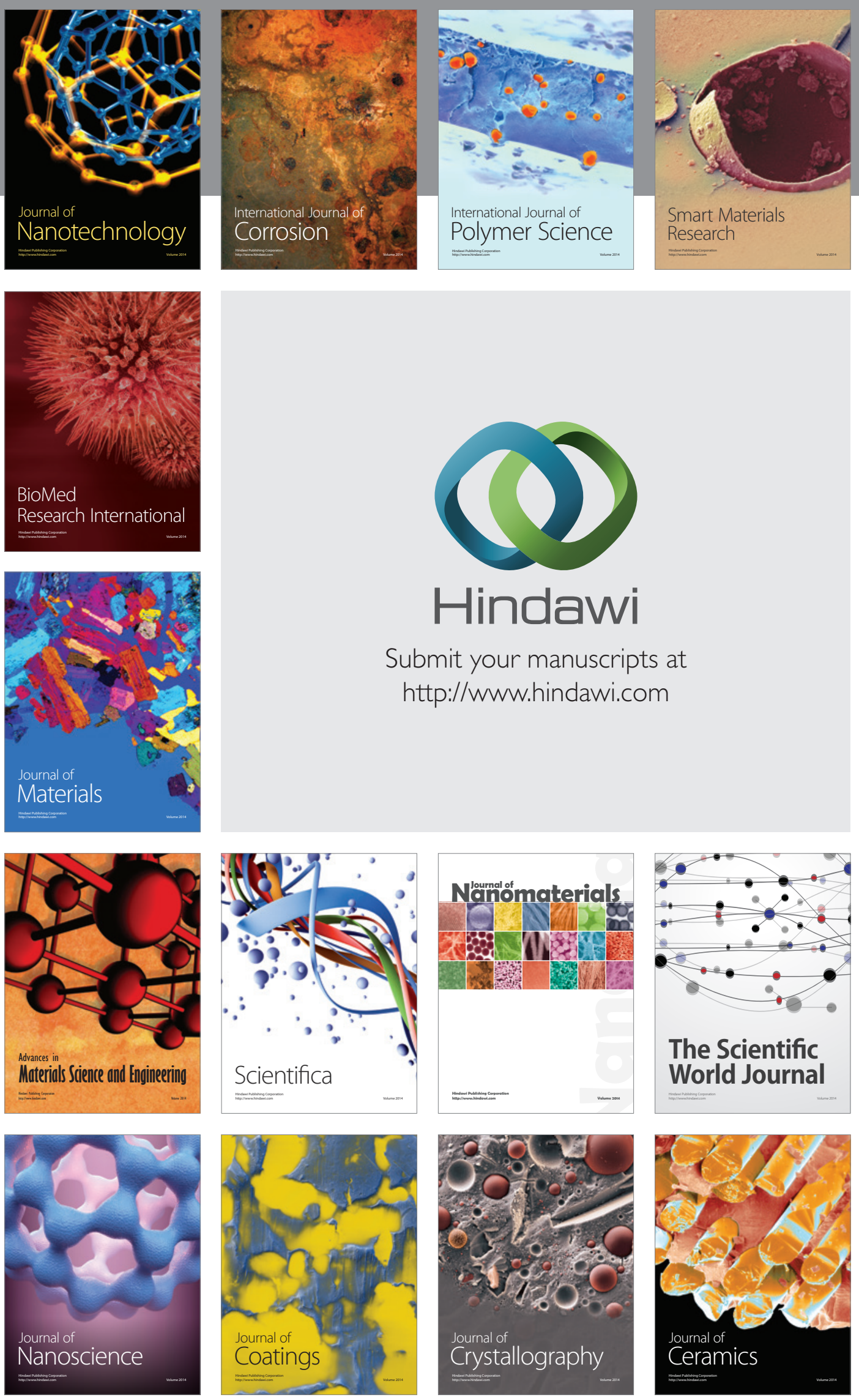

The Scientific World Journal

Submit your manuscripts at

http://www.hindawi.com

\section{World Journal}

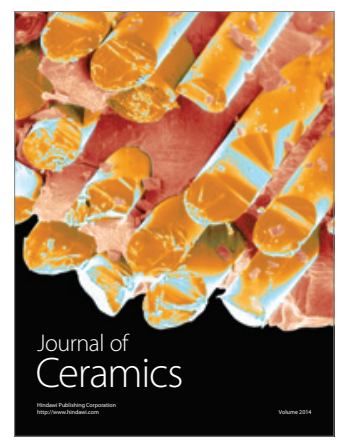

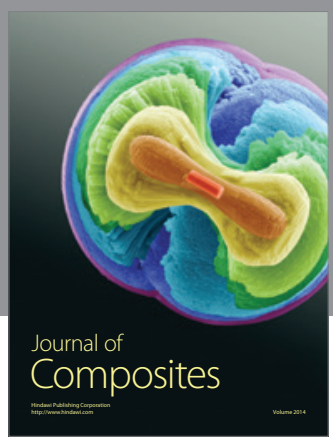
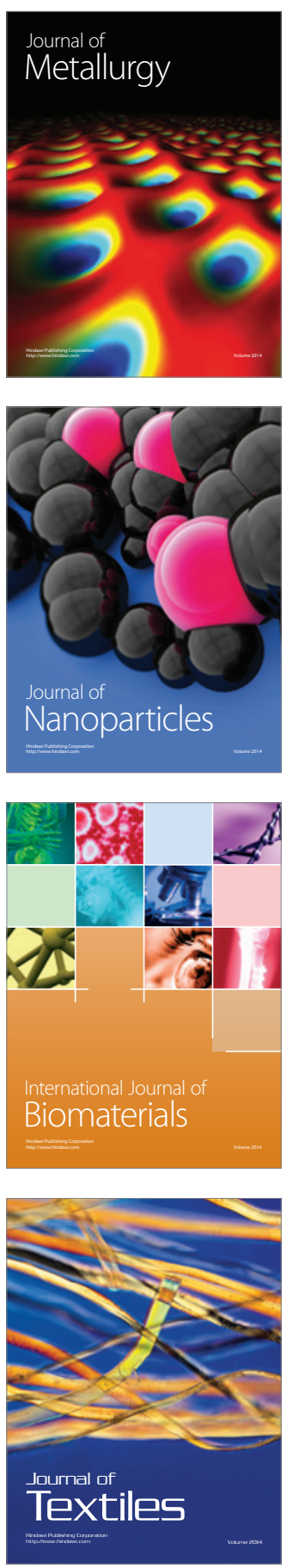